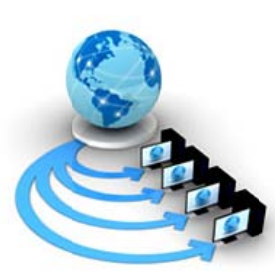

Volume 9, No. 1, January-February 2018

International Journal of Advanced Research in Computer Science

RESEARCH PAPER

\title{
A SURVEY OF RECENT TECHNIQUES FOR ENERGY COMPUTATION IN DISTRIBUTED COMPUTING NETWORKS
}

\author{
Kamlesh Kumar Verma \\ Department of Computer Science \\ Babasaheb Bhimrao Ambedkar University \\ Vidaya Vihar, Raebareli Road, Lucknow, India
}

\author{
Raj Shree \\ Department of Information Technology \\ Babasaheb Bhimrao Ambedkar University \\ Vidya Vihar Raebareli Road, Lucknow, India
}

\begin{abstract}
In recent years many techniques have been computed for energy consumption. The Energy Consumption is a very big challenging area for research in networks. There are various techniques for computing energy consumption in distributed computing as described in this paper. There is a different device attached to the distribution system for different electrical operation. They are Dynamic Power Management Techniques (DPMT) like as Dynamic Voltage Scaling (DVS), Dynamic Frequency Scaling (DFC), and Routing algorithm. In these techniques, Clock Gating technique is also a methodology but it is very expensive and effective. They consume very highly electricity working in operations. There are two major metrics for reducing energy consumption in networks as, power and time. So any reliable networks the performance must be less energy during throughput packet and transmission delivery. Energy Consumption is a valuable cost of any networks over the lifetime. In this paper the recent techniques used for energy optimization in distributed computing.
\end{abstract}

Keywords: Energy Consumption; Routing Algorithm; Dynamic Voltage Frequency Scaling (DVFS); Dynamic Power Management.

\section{INTRODUCTION}

Distributed Computing is an emerging area of research. The application of distributed computing devices power consumption is increasing day by day in all areas of network application throughout the world. The broadband wired communication network including the home personal computers and number of popular devices are based on the electrical application that gathered information from the surrounding. These devices are highly Energy Efficiency proposed due to their uses like Motherboard, processor, electromechanical devices and HDD in the computer for throughput the information processing. The proposed constraint makes energy consumption one of the most predicted areas among researcher and scientists. In these computing networks, there have been various techniques for reducing energy Consumption in previous and depend basically to traffic Load. The networks consume the largest amount of energy consumption which is observed to fix and variable in wired networks with a huge number of distributed networks. The highest energy consumption has seen in Dataware center and IP backbone networks. The method for reducing energy consumption is various types and matrices found for energy-related of networks. It's divided into main categories (1) Network workload. (2) The sleep mode approach. These two solutions can find the energy to save extra energy. Therefore, a technique to find for energy minimization or reducing is the research region in networks. The current work is focussed on computational techniques, which minimization the extra energy due to traffic load. The energy performance formulates as,

$$
\begin{gathered}
\text { Power } \propto \mathrm{CV}^{2} \times \mathrm{f} \\
\text { or } \\
\text { Power }=\mathrm{kCV}^{2} \times \mathrm{f}
\end{gathered}
$$

Where $\mathrm{C}$ is the capacitance, $\mathrm{V}$ is voltage, $\mathrm{f}$ is frequency and $\mathrm{k}$ is the load factor.
Performance-driven computer development has lasted decades. Computers have been developed higher performance. Three supercomputers have achieved Tera or Peta flops speed such as supercomputer: Cray Jaguar, Dawning Nebulae, and IBM Roadrunner

In the computing hardware characteristics as processing speed, memory/storage capacity, and network capacity. the power consumption an exponential speed, and the increased energy consumption in areas, Industry Impact, Environmental Impact, Research \& Development organization and Science and Technological Impact.

\section{METHODOLOGY}

Hammadi et al. [1] have described a survey on architectures and energy efficiency in data center networks. The survey of existing techniques approaches for energy saving and green data centers. Orgerie et al [2] have described the techniques to improve the energy performance of Computing and Networks devices in wired or wireless networks. They had described the solution to improve such as resource allocation, network traffic, and scheduling. Han Song et al. [3] have proposed an algorithm for macro-base stations and Femto-access points station for distributed computing. Moety Farah et al. [4] have proposed the multiple objective optimization methods is used in network power consumption. The minimization of transmission delay in wireless access networks used two techniques (i) IEEE 802.11 (ii) LTE network technology. Huang et al. [5] have given a model for minimizing the data redundancy for reliability to the distributed computing or cloud storage. Hassan A. et al. [6] have proposed a method for finding the scaling, communication, and performance for cloud datacenter. In this experiments, they found the point to point communication performance between nodes and network size. Agostini et al. [7] have proposed a method for the management of network and network performance 
technique for strategic multiplier networks. Alonso et al [8] have given a novel methodology for reducing power consumption in fat tree networks types. They have given a latency, routing algorithms methods. Zdraveski et al. [9] have proposed an algorithm for power saving by dynamic intelligent load balancing technique in distributed networks. Walkowiak [10] have developed the methodology for power saving. They have proposed for power saving two methods (i) elastic optical network (ii) operating expenditures for finding cost and power consumption in big data. Al-Ayyoub et al [11] have developed a model for power reducing in data centers. In this model, they have described the model in service like as (i) operational cost and (ii) service level agreement. Bilal et al [12] have described the various methods for finding the energy saving in the data center. Rengarajan et al. [13] have investigated the sleep modes technique for wireless access networks to the finding energy optimization. The fixed computing component in network utilization for finding the energy reduction. O'Neill et al. [14] have described the role of energy consumption for gaming system during the time of playing the game of the networking. The gaming architecture and designed in largescale distribution networks. Qiu et al. [15] have given a greedy model-based algorithm for improving the network robustness and energy reduction technique in networks. Cholda et al. [16] have described a solution to the find a state of art between energy efficiency and resilience in communication networks. A numerical algorithm is designed for finding the energy performance by iterative methods. Tang et al. [17] have given a particle swarm based optimization algorithm for finding the energy optimization. Jiang et al [18] have described a probability method for designing a reliable cloud data. In this method the securing the data and reliable to storage and re-outsourcing. Mershad et al [19] have given a mathematical model for analyzing the best result of power reducing in cloud data. They have good explained for power consumption in various computers hardware devices like CPU, Memory, and bandwidth. Alkhanak et al. [20] have described a review and classifications of $t$ Cost optimization scheme for scheduling in the cloud and distributed computing. He Li et al. [21] have discussed an algorithm for an interconnection network of high-performance computers. They have experimented algorithm through folded hypercube. Banditwattanawong et al. [22] have proposed a method for power optimization of intelligent cloud data centers networks. A. Lynch et al. [23] are designed to solve different kinds of distributed algorithms covered in this book. The algorithms that arise, the problems that telecommunications, information distribution, scientific computing and real-time process distribution. Elavarasan et al. [24] have proposed an algorithm for energy consumption in network infrastructure of the distributed data center. Lange et al [25] have discovered the method for reducing energy consumption in backbone networks data server. They have studied the improvements methods for the energy consumptions of all network operators. Bolla et al. [26] have studied the energy efficiency in future networks and energy aware of stable network architecture. Tseng and Chung et al. [27] have given the methods for energy consumption in IP network the energy consumption of mixed integer non-linear network optimization problem. Baliga et al [28] have analyzed of network distribution. They have described the energy consumption in wired, wireless, HFC networks, passive optical networks, WiMAX, and optical access networks. Gaona et al. [29] have proposed a model energy optimization on the design of hardware transactional memory systems. They have described the lazy-lazy network systems energy consumption. Chiaraviglio et al [30] have given a mathematical model for energy consumption in distributed networks. They have described the methodology in sleep mode, wake up and active mode. Castane et al. [31] have examined the experimental formula i.e. E-mc2 the framework model for energy modeling in cloud networks. Schien et al [32] have developed a mathematical model for energy consumption of online multimedia services at the time of variability. Lin et al. [33] have described mathematical model and algorithms for NP complete problem. They have solved NP problem by the method of Dijkstra's algorithm and Yen's k-shortest paths algorithm.

\section{A. Energy Saving Measurement}

- Simulator based power estimation likes NS2, OMNET++, OPNET, GloMoSim etc.

- Direct Measurement i.e. consolidated hardware devices.

- Event based estimation i.e. discrete time interval.

\section{B. Energy Computation in Hardware Devices of Distributed Networks}

The energy consumption consolidated hardware devices in data centre is shown figure 1 .

- Hard Disk: depend on different speed.

- Internet Card: it's depending on different speed.

- CPU: it's depending on different frequency and voltage.

- Motherboard: It's depending on voltage.

- RAM: It's depending on voltage

- Fan: It's depending on voltage.

- Processor Controller Board (PCB): It controls all process over voltage and power regulation.

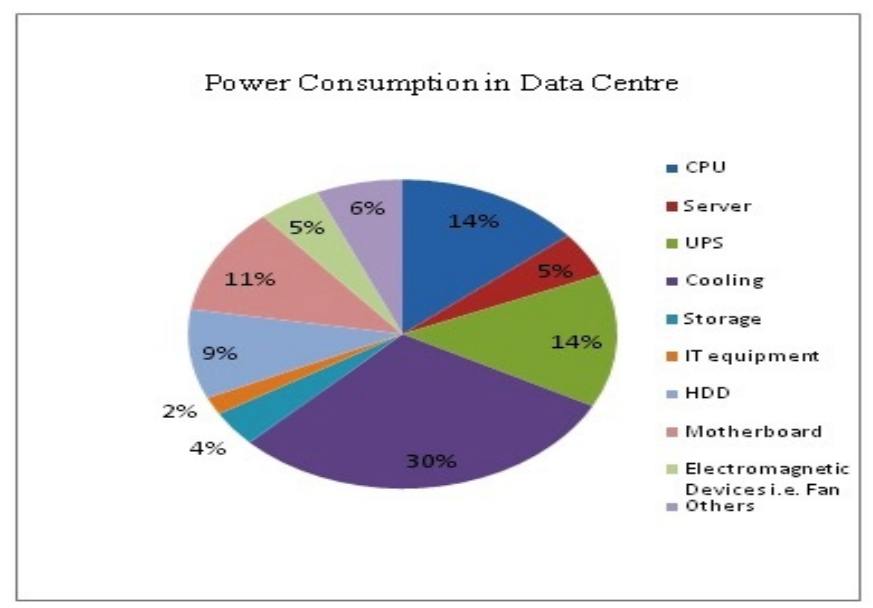

Figure1. Hardware Devices wise Energy Consumption in Data Centres. 


\section{MOdE OF ENERgy CONSUMPTION IN DISTRIBUTED NETWORKS}

\section{A. A Fixed Part or Static Mode Energy Consumption}

In this part depends on system size and computing type such as data storage, computing, and supporting networks elements. In that part the energy consumption occurs in leakage currents.

\section{B. A Variable or Dynamic Mode Energy Consumption}

In this part the works defining the results from the usage of computing, storage and network devices caused by system action. The energy consumption of a large scale distributed networks is a big challenge.

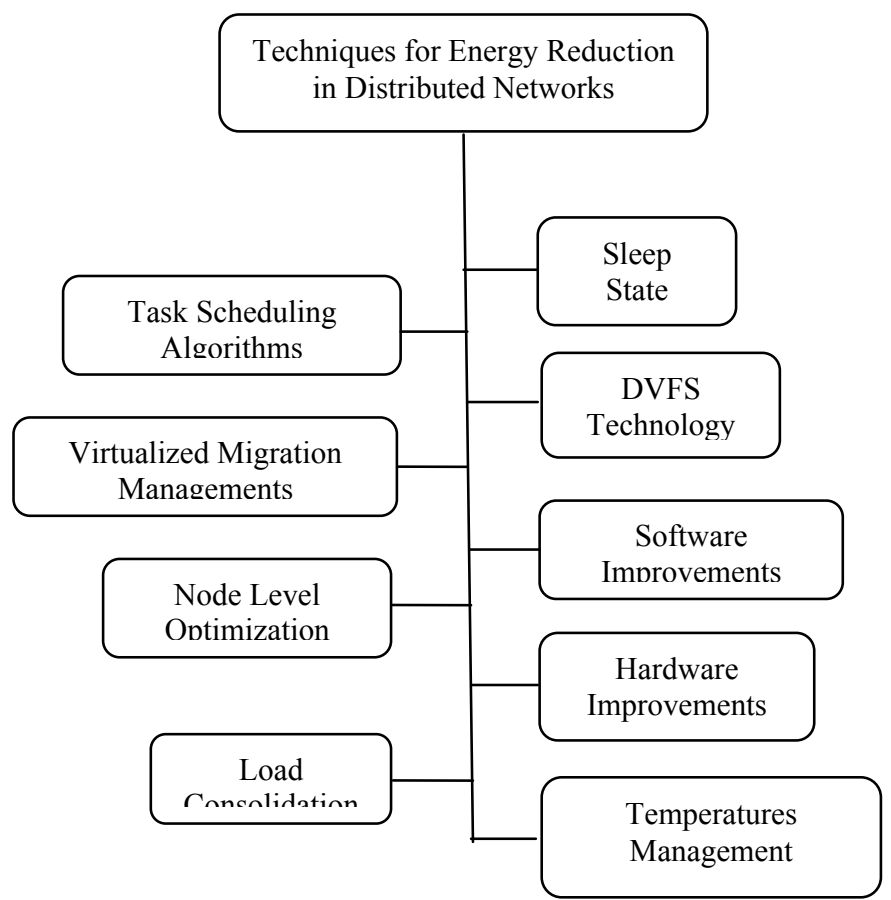

Figure 2. Energy Reduction Methodology in Distributed Computing.

\subsection{Computing Devices for Energy Efficiency}

Energy is defined as the rate of distributed system consumes electrical power during operation, Energy computations of the large scale distributed systems are as following techniques.

\section{DVS (Dynamic Voltage Scaling )}

Its method is used to conserve energy in a data centre that each processing elements like as,

Processing Peripheral Devices

$$
\sum \mathrm{PD}_{\mathrm{i}}=\mathrm{PD}_{1}+\mathrm{PD}_{2} \ldots \ldots \ldots \ldots \mathrm{PD}_{\mathrm{m}}
$$

Where peripheral devices like Motherboard, CPU, Hard Disk Drives (HDD). In that given all devices computes the formula. Like CPU energy consumption by Equation 2, here, supply voltage $\mathrm{V}$ can be scaled to a discrete number of voltage levels. Frequency reducing power consumption will increase the execution time of a task on a processing element. There are more useful for energy computation technique because the DVS approaches are (1) approximately solution of real problems (2) it has no switching (3) it has close relationship among voltage, power, and energy.

\section{DFS (Dynamic Frequency Scaling)}

DFS can be used for energy conservation by lowering the heat produced in a processor by the low frequency. The low frequency will increase the amount of time a PE needs to complete a task. Energy is conserved with DFS because the $\mathrm{PE}$ consumes less power when running at low frequency.

\subsection{Energy for Traffic Signal or Flow Data Packets}

The packet throughput of the transportation data for processing occurs energy signal exhibits. The energy of the signal in data transmission. The energy equation of every packet frames in data transmission is given below:

$$
E \int_{N_{0}}^{N_{n}}|x(\mathrm{t})| d t
$$

the stationary equation of the data forwarding as given Equation 5.

$$
y=x(\mathrm{t})=a(\sin \omega t+\phi)
$$

Where $x(t)$ is linear time with distance node from vertices or destination point. a is the amplitude of the transmission time, $\omega$ is an angular frequency which depends on cycle per second $\omega=2 \pi f, f=\frac{1}{T}$, and during data sending, $\varphi$ is the phase difference between from the packet source to destination packet. If $\mathrm{x}(\mathrm{t})$ is the voltage applied across a load $\Omega$ resistor, then $\mathrm{x}(\mathrm{t})$ is the power estimation. The integration of power over time is total energy of the signal is called energy $\mathrm{E}$ that can be extracted from the signal continuous time $\mathrm{T}$ and the total energy depending on the time interval.

\section{POWER Computation TeChNiQues}

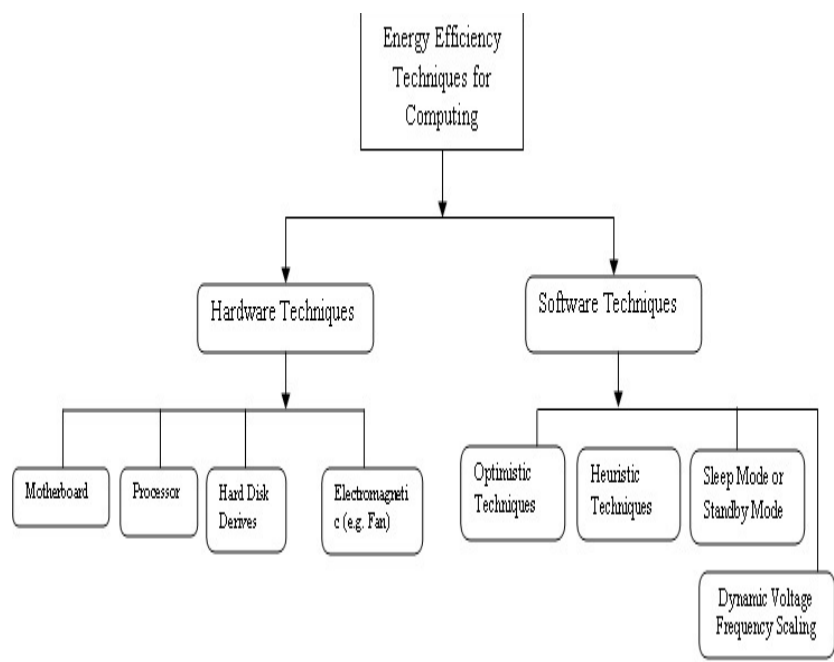

Figure 3: Energy Efficiency Computing Model

\section{A. Distributed and Data Centre Power Mnagement}

The Following diagram represented in given below: 
- Temperature Control by Data Centre Air Condition (DCAC)

- Energy aware of task scheduling.

- Load estimation.

- System configuration energy

- Energy emits with $\mathrm{CO}_{2}$.

- Load balancing through data centres.

\section{ENERgY COMPUTATION BY ROUTING A LGORITHMS}

There are various methods for finding the energy consumption by the method of routing algorithms.

\section{A. Bellman- Ford Algorithm.}

Its algorithms solve the single source shortest paths problem. The graphs where the edge-weights may be negative, but no negative weight cycle exists in dynamic programming. The Bellman-ford equation is

$$
d_{x}(y)=\min _{v}\left\{c(x, v)+d_{v}(y)\right\}
$$

$d_{x}(y)$, be the cost of the least-cost path from node $x$ to node $y$. where the min $\mathrm{v}$, in the equation, is taken over all of $\mathrm{x}$ is the neighbors traveling from $\mathrm{x}$ to $\mathrm{v}$, if we take the least-cost path from $\mathrm{v}$ to $\mathrm{y}$, the path cost will be $\mathrm{c}(\mathrm{x}, \mathrm{v})+\mathrm{d}_{\mathrm{v}}(\mathrm{y})$. It begin by traveling to some adjacent $\mathrm{v}$, the minimum cost from $\mathrm{x}$ to $\mathrm{y}$ is of $\mathrm{c}(\mathrm{x}, \mathrm{v})+\mathrm{d}_{\mathrm{v}}(\mathrm{y})$ across whole near vertices $\mathrm{v}$.

\section{B. Optimization Scheduling Algorithms for Energy}

Efficient Networks The optimization algorithms are used to find solutions for the energy consumption through task scheduling allocation problem in the following figure 4 . is represented:
(1) Link sates routing

(2) Distance vector routing

(3) Minimum, Maximum and Mini-Max Scheduling

\section{Dijkstra's Algorithm}

In this algorithm shortest path from a single source to all destinations i.e. vertices on a graph with non-negative weights. The algorithm is used for single source shortest path but no negative cycles. In this algorithms divide the nodes into two sets (a) Provisional Method (b) Permanent Method. It finds the neighbors of a current node makes them provisinal and examines if they pass the criteria makes them permanent. Dijkstra's algorithm requires processing time proportional to the number of links in the graph i.e. $\mathrm{n} * \mathrm{k}$ links and describes by the log of the number of nodes in the net, it defines the originated term as $\mathrm{O}(\mathrm{n} * \mathrm{k} \log n)$.

\section{Conclusions}

Energy consumption is one of the most important roles in the wired and wireless sensor networks. The main objective of energy consumption computing is two types (i) Softwarebased energy consumption techniques (ii) Hardware-based energy consumption techniques. The DVFS techniques to reduce power consumption of CPU during communication, The routing scheduling techniques and algorithms are methods of finding energy consumption as an important computational method in networks. The Energy saving in wire or distributed networks having a lot of challenges across the world. In this paper, the energy consumption not only for energy consumption but it is also used for energy balance to increase and lifetime for futuristic.

\section{Optimization Scheduling Algorithms -}

The routing algorithms in scheduling as

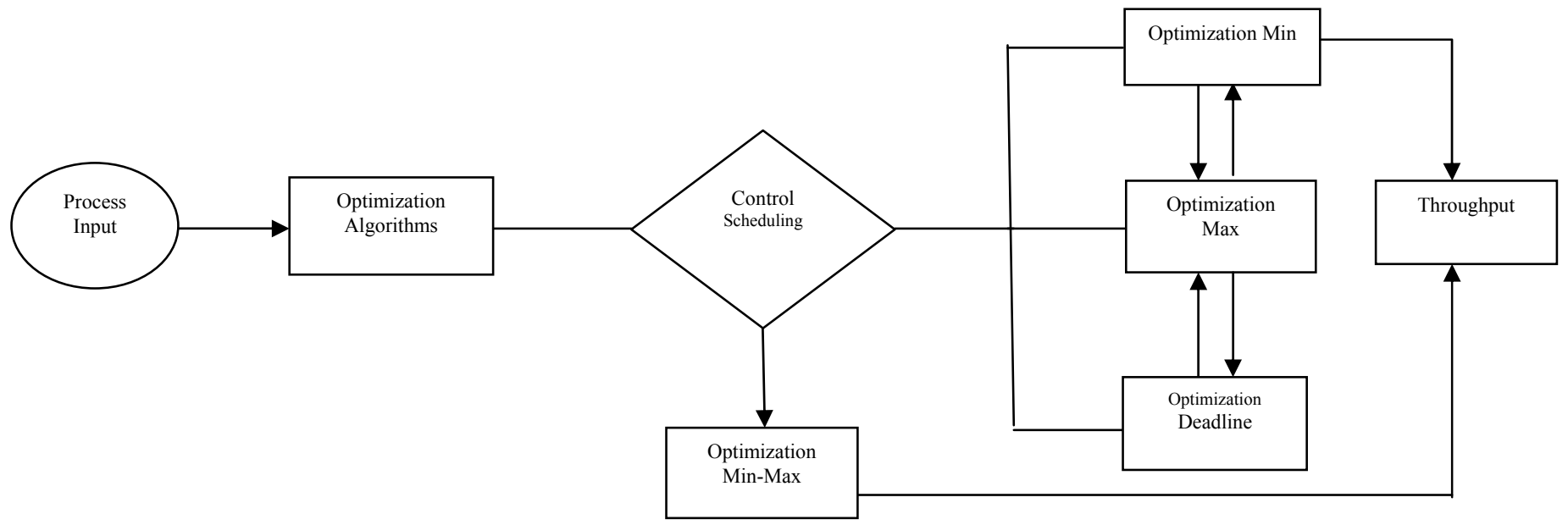

Figure 4. Flowchart of Optimization Scheduling Algorithms in Networks 


\section{REFERENCES}

[1] Hammadi, Ali., and Mhamdi, Lofti., "A Survey On Architectures and Energy Efficiency in Data Center Networks",Computer Comm, Science Direct, Elsevier https://doi.org/10.1016/j.comcom.2013.11.005, pp. 1-21, 2014.

[2] Orgerie, Anne-Cecile., De Assuncao, Marcos Dias., et al."A Survey on Techniques for Improving The Energy Efficiency of Large Scale Distributed Systems", ACM Computing Surveys, pp. 1-35, 2014.

[3] Hn, Song. Li., Xin, bin., Liu, Zhixin., and Guan, Xinping.," Distributed Hierarchical Game-Based Algorithm for Downlink Power Allocation in OFDMA Femto Cell Networks".Computer Networks, pp 1-13, 2015.

[4] Moety,Farah., Lahoud, Samer., Cousin,Bernard., and Khawam, Kinda.," Optimization Models for the Joint Power - Delay Minimization Problem in Green Wireless Access Networks." Computer Networks 92 148167,http://dx.doi.org/10.1016/j.comnet.09.032, 2015.

[5] Huang, Zhen., Chen, Jinbang., Lin, Yisong., You, Pengfei., and Peng, Yuxing.,"Minimizing Data Redundancy for High Reliable Cloud Storage Systems". Computer Networks pp. 164-177, 2015.

[6] A.,Hassan., Hanan, A., Mohamed, Shimaa., M Sheta, Walaa., "Scalability and Communication Performance of HPC on Azure Cloud". Egyptian Informatics Journal, 2015.

[7] Agostini n, Lara. Filippini, Roberto. Nosella, Anna., "Management and Performance of Strategic Multipartner SME Networks". International Journal Production Eco. pp. 376-390, 2015.

[8] Alonso,M., Coll, S., Martínez ,J.M., Santonja,V.,P, López.," Power Consumption Management in Fat-Tree inter Connection Networks". Parallel Computing pp. 5980, 2015.

[9] Zdraveski, Vladimir., Todorovski, Mirko., Kocarev, Ljupco., " Dynamic Intelligent Load Balancing in Power Distribution Networks". Electrical Power and Energy Systems pp 157-162, 2015.

[10] Krzysztof, Walkowiak., Michał, Woźniak., Mirosław, Klinkowski., Wojciech, Kmiecik., "Optical Networks for Cost-Efficient and Scalable Provisioning of Big Data Traffic". Inter J. of Parallel, Emergent and Distributed Systems, Vol. 30, No. 1, pp. 15-28, 2015.

[11] Al-Ayyoub, Mahmoud., et al.“Optimizing Expansion Strategies for Ultrascale Cloud Computing Data Centers." Simulation Modelling Practice and Theory pp. 15-29, 2015.

[12] Bilal,Kashif.,Khalid,Osman.,Alvarez,Enrique.,ameed,Abdu 1., et, al., "A Taxonomy and Survey On Green Data Center Networks". Future Generation Computer Systems, pp. 189-208, 2014.

[13] Rengarajan, Balaji., Rizzo, Gianluca., Ajmone Marsan, Marco.,"Energy-Optimal Base station Density in Cellular Access Networks with Sleep Modes". Computer Networks, pp. 152-163, 2015.

[14] O’Neill, Danielle, and Hook, Alan., "Future Energy Networks and the Role of Interactive Gaming As Simulation". Computer Networks, pp. 01-11, 2016.
[15] Qiu, Tie., Luo, Diansong., Xia, Feng., et. al. "A Greedy Model with Small World for Improving the Robustness of Heterogeneous Internet of Things", Computer networks, pp. 01-17, 2016.

[16] Chołda, Piotr., and Jaglarz, Piotr., "Optimization Simulation - Based Risk Mitigation in Resilient Green Communication Networks", Journal of Network and Computer Applications 59 pp. 134-157, 2016.

[17] Tang, Meiqin., and Xin, Yalin., "Energy Efficient Power Allocation In Cognitive Radio Network Using Coevolution Chaotic Particle Swarm Optimization”. Computer Networks, pp. 1-11, 2016.

[18] Jiang, Tao., et al. "Towards Secure and Reliable Cloud Storage against Data Re-Outsourcing". Future Generation Computer Systems, pp 86-94, 2015.

[19] Mershad N, Khaleel., Artail, Hassan., Saghir, Mazen., Hajj, Hazem., Awad, Mariette., "A Mathematical Model to Analyze the Utilization of a Cloud Data Center Middleware",Journal of Network and Computer Applications, pp. 399-415, 2016.

[20] Alkhanak, EhabNabie., Peck Lee,Sai., Rezaei, Reza., Parizi, Reza Meimandi., "Cost Optimization Approaches for Scientific Work Flow Scheduling in Cloud and Grid Computing : A Review, Classifications and Open Issues". The Journal of Systems. and Software, pp. 1-26, 2016.

[21] He, Li., and Li, Furong., "A Fast Diagnosis Algorithm for Interconnection Network in High Performance Computers". International Journal of Parallel, Emergent and Distributed Systems, pp. 34-46, 2016.

[22] Bandit wattanawong, Thepparit. ,Masdisornchote, Masawee., thayopas, Putchong., "Multi-Provider Cloud Computing Network Infrastructure Optimization". Future Generation Computer Systems, pp. 116-128, 2016.

[23] A. Lynch, Nancy.,"Distributed Algorithms(The Morgan Kaufmann Series in Data Management Systems)", publisher: Morgan Kaufmann, ISBN:1558603484, 9781558603486, 1996.

[24] Elavarasan, R., and Steffi, Perpectual A.,"Energy Efficient Data Collections Structure for Wireless Sensor Networks". International Journal of Science and Research (IJSR), ISSN 2319-7064, Volume 3, Issue 4, April 2014.

[25] Lange,C.,Kosiankowski, D., Weidmann, R., Gladisch, A., "Energy Consumption of Telecomm Networks and Related Improvement Options".IEEE Journal of Selected Topics in Quantum Electronics, 17(2), pp.285- 295, 2011.

[26] Bolla, R.,et al. "Energy Efficiency In The Future Internet a Survey of Existing Approaches and Trends In Energy- Aware Fixed Network Infrastructures". IEEE Comm. Surveys \& Tutorials, 13(2), pp.223- 244, 2011.

[27] Tseng, Po-Kai., and Chung, Wei-Ho., "Near Optimal Link On/Off Scheduling and Weight Assignment for Minimizing IP Network Energy Consumption". Computer Communications 35.6:729-737, 2012.

[28] Baliga, Jayant., Ayre, Robert., Hinton, Kerry., and S.Tucker, Rodney., "Energy Consumption In Wired and Wireless Access Networks", Energy Efficiency in Communication".IEEE Communication Magazine 49.6 : pp.70-77, 2011.

[29] Gaona, E., Titos, R., Fernández, J., Acacio, M. E., 
Fernández, J., "On The Design of Energy Efficient Hardware Transactional Memory Systems", Concurrency 2012 and Computation: Practice and Experience, Published,

[30] Chiaraviglio, Luca., et al. "Modelling Sleep Mode Gains In Energy-Aware Networks". pp. 051- 3066, Computer Networks, 2013.

[31] Castañé, Gabriel G., Llopis, Pablo., and Carretero,Jesús., “ E-Mc2: A Formal Framework For Energy Modelling In Cloud Computing". Simulation Modelling Practice and Theory", pp. 56- 75, 2013.
[32] Schien, Daniel., Shabajee, Paul., et al., "Modelling and Assessing Variability in Energy Consumption During the Use Stage of Online Multimedia Services". Journal of Industrial Ecology Information and Communication Technology (ICT), 2013.

[33] Lin, Gongqi., Soh, Sieteng., Chin, Kwan-Wu., Lazarescu, Mihai., "Efficient Heuristics For Energy-Aware Routing In Networks with Bundled Link". Computer Networks pp. 1774-1788, 2013. 